\title{
Spatiotemporal Coordination of Slow-Wave Ongoing Activity across Auditory Cortical Areas
}

\author{
Brandon J. Farley and Arnaud J. Noreña \\ Laboratory of Adaptive and Integrative Neuroscience, CNRS, and Aix-Marseille Université, Fédération de Recherche 3C, 13331 Marseille, France
}

\begin{abstract}
Natural acoustic stimuli contain slow temporal fluctuations, and the modulation of ongoing slow-wave activity by bottom-up and top-down factors plays essential roles in auditory cortical processing. However, the spatiotemporal pattern of intrinsic slow-wave activity across the auditory cortical modality is unknown. Using in vivo voltage-sensitive dye imaging in anesthetized guinea pigs, we measured spectral tuning to acoustic stimuli across several core and belt auditory cortical areas, and then recorded spontaneous activity across this defined network. We found that phase coherence in spontaneous slow-wave (delta-theta band) activity was highest between regions of core and belt areas that had similar frequency tuning, even if they were distant. Further, core and belt regions with high phase coherence were phase shifted. Interestingly, phase shifts observed during spontaneous activity paralleled latency differences for evoked activity. Our findings suggest that the circuits underlying this intrinsic source of slow-wave activity support coordinated changes in excitability between functionally matched but distributed regions of the auditory cortical network.
\end{abstract}

\section{Introduction}

Ongoing slow-wave activity plays an integral role in cortical sensory processing. The state of ongoing activity determines excitability, influencing cortical responses to sensory stimuli as well as perception (Arieli et al., 1996; Petersen et al., 2003b; Lakatos et al., 2005; Busch et al., 2009; Stefanics et al., 2010; Ng et al., 2012). In the auditory cortex, slow-wave activity tracks the temporal dynamics of natural acoustic stimuli, which themselves have important slow temporal fluctuations (Luo and Poeppel, 2007; Kayser et al., 2009; Chandrasekaran et al., 2010; Szymanski et al., 2011). Further, internal factors can modify slow-wave activity patterns. Attending to a rhythmic stimulus alters the phase of ongoing slow-wave activity or its temporal coherence (Lakatos et al., 2008; Elhilali et al., 2009; Xiang et al., 2010). This intrinsic modulation is proposed to enhance the representation of a specific relevant sound in the presence of competing sounds or noise (Schroeder and Lakatos, 2009; Shamma et al., 2011).

Despite the central role of ongoing slow-wave activity in auditory processing, the spatiotemporal properties of intrinsic sources of this activity are unknown. The pattern of ongoing activity and its relationship with neuronal tuning (Kohn et al., 2009; Ringach, 2009) can inform the underlying circuits of the

Received 0ct. 30, 2012; revised Dec. 15, 2012; accepted Dec. 23, 2012.

Author contributions: B.J.F. and A.J.N. designed research; B.J.F. performed research; B.J.F. and A.J.N. analyzed data; B.J.F. and A.J.N. wrote the paper.

The authors declare no competing financial interests.

This work was supported by the Tinnitus Research Initiative and by L'Agence Nationale de la Recherche Grant ANR-2010-JCJC-1409-1. The authors wish to thank Wen-Jie Song, Nicolas Catz, Christian Xerri, Yoh'iZennou-Azogui, Ali Gharbi, Sebastian Roux, and Frederic Chavane for technical assistance; as well as Jos Eggermont and anonymous reviewers for valuable comments on a previous version of the manuscript.

Correspondence should be addressed to Brandon J. Farley, Laboratory of Adaptive and Integrative Neuroscience, CNRS, and Aix-Marseille Université, Fédération de Recherche 3C, 3 Place Victor Hugo, 13331 Marseille, France. E-mail: brandon.farley@univ-provence.fr.

DOI:10.1523/JNEUROSCI.5079-12.2013

Copyright $\odot 2013$ the authors $\quad 0270-6474 / 13 / 333299-12 \$ 15.00 / 0$ activity, as well as its potential role in modulating auditory processing. Observations in other modalities indicate that in the absence of sensory stimulation, subthreshold slow-wave events are diffuse or take the form of traveling waves across cortical fields (Volgushev et al., 2006; Ferezou et al., 2007; Crunelli and Hughes, 2010; Harris and Thiele, 2011). These observations suggest that slow-wave activity can synchronize processing over distributed regions, but not in a specific or neuronal tuning-dependent manner. On the other hand, recordings of high-gamma $(60-250 \mathrm{~Hz})$ band or multiunit activity in the auditory cortex have revealed spontaneous activity patterns that relate to patterns of neuronal tuning (Brosch and Schreiner, 1999; Fukushima et al., 2012). Given the known relationship between slow-wave phase and multiunit amplitude (Lakatos et al., 2005), these observations raise the possibility that specific patterns could also be present in ongoing slow-wave activity of auditory cortex.

Addressing the properties of ongoing activity throughout the auditory cortex requires a technique capable of sampling across a wide field but with high spatial and temporal resolution. We show that voltage-sensitive dye imaging (Shoham et al., 1999; Petersen et al., 2003a) can reveal spontaneous and stimulusdriven ongoing activity across a distributed network of several tonotopically defined cortical fields. We find highly structured patterns in ongoing slow-wave activity, implicating the involvement of long-range circuits that can support spatially and temporally coordinated modulation of intrinsic excitability across the network.

\section{Materials and Methods}

Surgical procedures. Experimental protocols followed the guidelines of French and European laws on laboratory animal care and use, and were approved by the Animal Care Committee of Bouches-du-Rhones, France. The data from this study come from 15 male and female adult guinea pigs (Dunkin Hartley breed from Charles River Laboratories). Immediately before surgery, each animal was checked for the presence of 
A

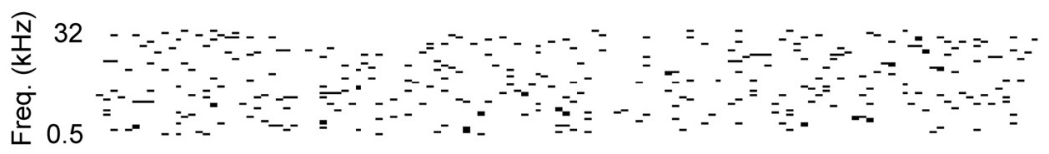

B

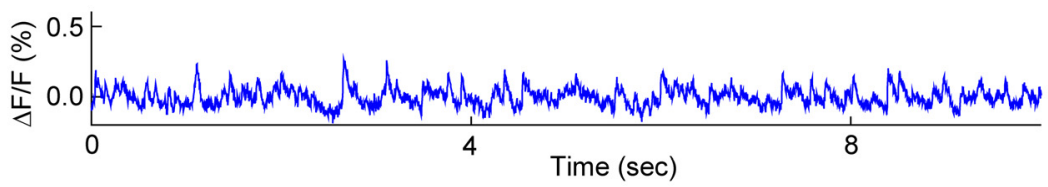

C

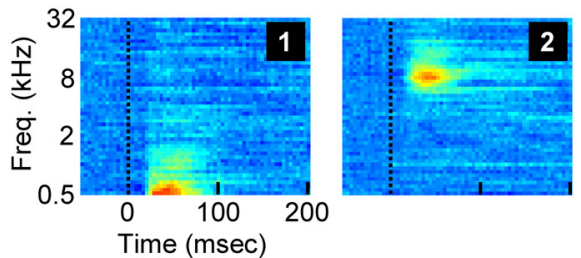

D
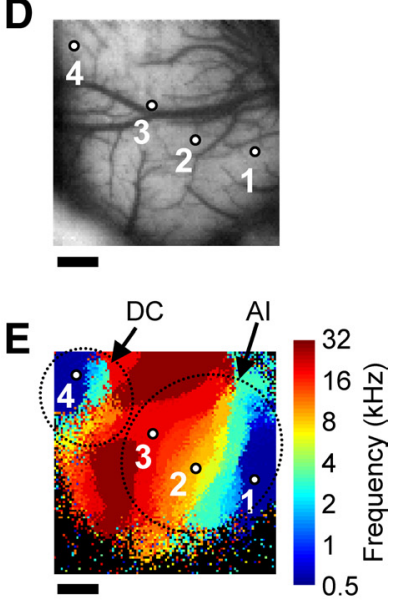

Figure 1. Ongoing activity recorded during continuous acoustic stimulation reveals acoustic frequency tuning across auditory cortex core areas. $A$, Schematic of sound stimulus, composed of pseudo-randomly occurring pure-tone pips with frequencies ranging from $500 \mathrm{~Hz}$ to $32 \mathrm{kHz}$. B, Ongoing fluorescence signal measured during acoustic stimulation from a small region ( $2 \times 2$ pixels, centered on region " 1 ") of Al. C, Acoustic frequency-receptive fields from example ( $2 \times 2$ pixel) regions of core auditory cortex areas Al and DC, showing the averaged temporal response to each of the 49 different frequencies. $\boldsymbol{D}$, Image of cortical vasculature within the $5 \times 5 \mathrm{~mm}$ imaging area, with the four example region locations indicated. $\boldsymbol{E}$, Each pixel is color-coded according to which of the 49 frequencies elicited the maximal response, yielding a tonotopic map of auditory cortex fields Al and DC. Nonresponsive pixels are colored black. Dotted lines show approximate borders of Al and DC for illustrative purposes only. Scale bars: D, E, $1 \mathrm{~mm}$.

a bilateral pinnal reflex in response to sound. Animals were pretreated with atropine, and anesthesia was induced and maintained with a mixture of ketamine ( $46 \mathrm{mg} / \mathrm{kg}$ ) and xylazine $(24 \mathrm{mg} / \mathrm{kg})$, with supplemental doses at half this concentration each hour. The analgesic Tolfedine was also administered, and lidocaine was applied to incision points. Body temperature was maintained at $37^{\circ}$. A tracheotomy was performed, and animals were artificially respirated with air containing concentrated oxygen. Anesthesia depth was monitored by EKG rate, expired $\mathrm{CO}_{2}$ levels, and lack of response to paw pinch. Animals were immobilized by a metal piece cemented to the skull, which could be fixed to a stereotaxic instrument. A craniotomy and durotomy were performed over the region of auditory cortex. The voltage-sensitive dye RH-1691 (Optical Imaging) was applied by soaking a piece of absorbent foam in dye solution $(0.5$ $\mathrm{mg} / \mathrm{mL}$ in ACSF) and placing this over the cortical surface for $2 \mathrm{~h}$. After rinsing, agarose ( $1.5 \%$ in saline) was applied over the stained cortex, and a transparent coverslip was used to seal the craniotomy and prevent excess brain movement.

Acoustic stimulation. Experiments were performed within an acoustically insulated sound booth. Acoustic stimuli were produced by a Tucker-Davis Technologies (model RP2) acoustic generator and delivered through a calibrated Sennheiser headphone (model HD595) placed $10 \mathrm{~cm}$ from the contralateral ear. For deriving acoustic frequencyreceptive fields and resulting tonotopic maps for the auditory cortex core fields primary auditory cortex (AI) and dorso-caudal cortex (DC), the "multitone" stimulus (deCharms et al., 1998) consisted of 50-msduration pure tones of 49 different frequencies (from $500 \mathrm{~Hz}$ to $32 \mathrm{kHz}$, with eight frequencies per octave) each presented randomly over time (Fig. 1A). The interstimulus intervals for tones of each frequency were Poisson distributed with an average interval of $500 \mathrm{~ms}$, and with intervals $<50 \mathrm{~ms}$ not permitted. Consequently, the average interstimulus interval considering tones of all frequencies was $\sim 10 \mathrm{~ms}$. Moreover, because the presentation times for each tone were independently determined, tones of different frequencies could overlap in time. All pure tones had a gamma envelope with maximal amplitude at $8 \mathrm{~ms}$ and were presented at $70 \mathrm{~dB}$ SPL. The sequence of tones lasted $180 \mathrm{~s}$ in total, with $30 \mathrm{~s}$ of this sequence played at a time with concurrent data acquisition (60 s pauses were placed between each acquisition epoch). Alternatively, for experiments requiring identification of individual belt auditory cortical areas, an isolated pure-tone protocol was preferable, and was used to generate single tone-evoked and tonotopic maps. In this case, the stimulus was a $50 \mathrm{~ms}$ pure tone (one of seven frequencies, in one-octave steps, ranging from $500 \mathrm{~Hz}$ to $32 \mathrm{kHz}$ ) played in isolation, the trials were separated by $6 \mathrm{~s}$ silent intervals, and pure-tone stimulus trials were interleaved with blank trials.

Data acquisition and calculation of stimulus-evoked responses. During data acquisition, the brain was illuminated with an epi-illumination stage including an excitation filter of $610 \mathrm{~nm}$. We used a dichroic mirror of 650 $\mathrm{nm}$ and an exit filter of $665 \mathrm{~nm}$. The light path was blocked in between acquisition epochs by a shutter. We employed the MiCAM ULTIMA (SciMedia) imaging and data-acquisition system, which uses a CMOS (complementary metal-oxide-semiconductor) sensor with $100 \times 100$ imaging elements. Two different camera lens configurations permitting two different magnifications were used, which allowed for sampling from either a $5 \times 5 \mathrm{~mm}$ or $10 \times 10 \mathrm{~mm}$ extent of the cortical surface. The sampling rate was $250 \mathrm{~Hz}$ for all experiments. For all ongoing activity measurements (during silence or during acoustic stimuli), each acquisition epoch consisted of 8192 frames $(\sim 32 \mathrm{~s})$ with a $60 \mathrm{~s}$ pauses in between. For ongoing activity measurements, artifacts related to EKG and respiration were removed using previously established methods (Lippert et al., 2007). Briefly, the time of each EKG and respiration event was automatically detected and recorded throughout the experiment. This permitted calculating an average "EKG-triggered artifact" or "respiration-triggered artifact" for each pixel. The average artifact was then subtracted from the original data traces at each EKG and respiration occurrence. Such artifacts were generally small within the auditory cortical region. For isolated pure-tone experiments, 256 frames surrounding the presentation of each tone were collected, during pauses in the respiration. In each trial, a pure-tone stimulus (or a "blank" stimulus) was presented at a constant phase of the EKG. Subsequently, common artifacts related to EKG or respiration could be removed by subtracting the averaged response to interleaved blank stimuli from responses to pure tones. All data are displayed as the percentage change in fluorescence over the baseline fluorescence value for each pixel $(\Delta F / F)$.

Receptive fields were derived individually for each pixel. For experiments using the multitone stimuli, the averaged response time locked to the presentation of each of the 49 frequencies was calculated. The "best frequency" of the pixel was defined as the frequency eliciting the maximal response, across all frequencies and time points. The best frequency was similarly defined for isolated pure-tone stimuli. Tonotopic maps were created by color coding each sound-responsive pixel according to its best frequency, with nonresponsive pixels colored black. Sound-responsive pixels were defined as those that responded significantly to a pure tone within the first $100 \mathrm{~ms}$ after stimulation, following statistical correction for multiple tones and response latencies. 
A

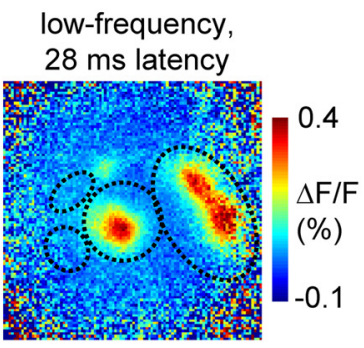

B

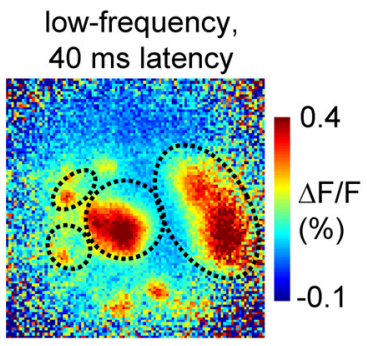

C

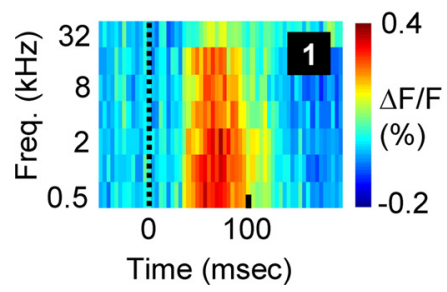

D

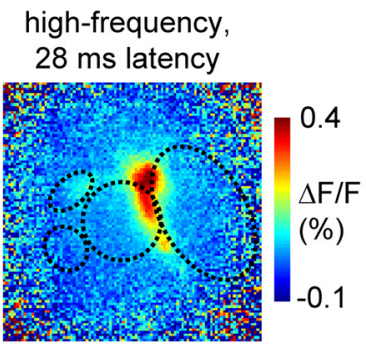

E

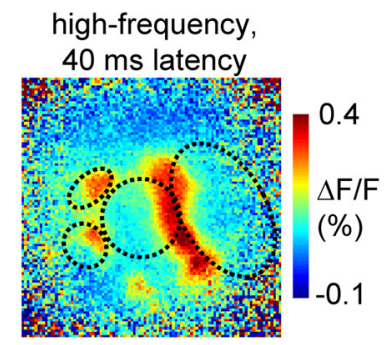

$\mathbf{F}$

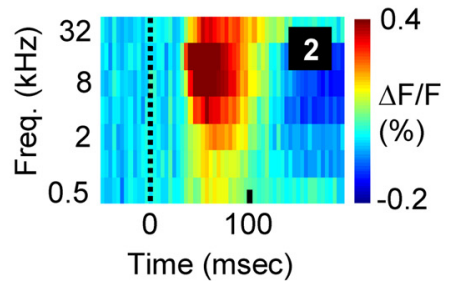

G

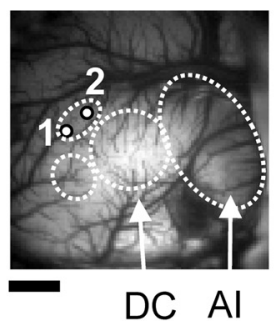

H

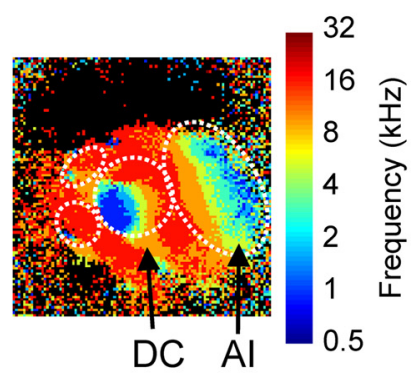

I

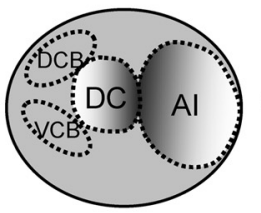

Figure 2. Cortical activation pattern in response to sparsely presented stimuli reveals organization of core and belt auditory cortical areas. $\boldsymbol{A}, \boldsymbol{B}$, Cortical activation pattern following stimulation with a low-frequency $(0.5 \mathrm{kHz})$ tone. At a latency of $28 \mathrm{~ms}(\boldsymbol{A})$, core areas $A \mathrm{I}$ and $D C$ are activated. At a longer latency of $40 \mathrm{~ms}(\boldsymbol{B})$, belt regions are also recruited. $\boldsymbol{C}$, $A$ coustic frequency-receptive field from the example $(2 \times 2$ pixel) region of the DCB area (region " 1 " indicated in $\boldsymbol{G})$, showing the averaged temporal response to each of the seven sparsely presented pure tones. $\boldsymbol{D}, \boldsymbol{E}$, Activation pattern following high-frequency ( $32 \mathrm{kHz}$ ) tone stimulation at a latency of $28(\boldsymbol{D})$ or $40 \mathrm{~ms}(\boldsymbol{E})$. $\boldsymbol{F}$, Acoustic receptive field from the example region of the DCB area (region " 2 " indicated in $\boldsymbol{G}$ ), showing the averaged temporal response to pure tones of seven different frequencies. $\mathbf{G}$, Image of vasculature for the $10 \times 10 \mathrm{~mm}$ imaging area, with the two example region locations of $D C B$ indicated. $\boldsymbol{H}$, The tonotopic map derived by color coding each pixel according to the frequency eliciting the maximal response. $I$, Schematic illustration of guinea pig auditory cortex, showing the two core fields Al and $D C$ and the two surrounding belt fields that were the focus of this study. Scale bar: $\boldsymbol{G}$ (for $\boldsymbol{A}, \boldsymbol{B}, \boldsymbol{D}, \boldsymbol{E}, \boldsymbol{H}), 2 \mathrm{~mm}$.

For computing the evoked-activity response latency for each auditory cortical area, we fitted a sigmoid function to the rising portion of the evoked response measured in single trials in response to an isolated puretone stimulus. The first time at which this fitted response reached 3 SDs above the prestimulation baseline was defined as the response latency for that trial, and the mean over all trials was calculated.

Definition of tonotopic regions within core and belt areas. For multiple analyses in this study (indicated in the text), pixels in core auditory cortex areas AI and DC were grouped into six bins based on their best frequency, derived from the acoustic frequency-receptive field. Bins were composed of pixels having best frequencies of $0.5-1,1-2,2-4,4-8,8-16$, or $16-32 \mathrm{kHz}$. The " $16-32 \mathrm{kHz}$ bin" comprised pixels of both AI and DC. Within each bin, we calculated the mean response over all pixels at each time point.

The auditory belt regions included in this study were the ventro-caudal belt (VCB) area and the dorso-caudal belt (DCB) area, and were identified as punctate regions of activation in response to pure-tone stimuli, which were caudal to and spatially segregated from activation in the DC area (Fig. 2). In addition, these belt areas were characterized by having longer evoked-activity response latencies than the core areas (compare Figs. 2, 7). These criteria were based on those defined in earlier electrophysiological studies of guinea pig auditory cortex (Redies et al., 1989b; Wallace et al., 2000). We identified the pixel in the VCB or DCB region that exhibited the earliest-latency response to a low-frequency $(0.5 \mathrm{kHz})$ or high-frequency $(32 \mathrm{kHz})$ tone. A $2 \times 2$ pixel region including this pixel was defined as the low- or high-frequency region of the corresponding belt area. The low- and high-frequency regions were spatially offset from one another within these caudal belt areas. Following the calculation of the phase-locking vector or phase differences, data from the VCB and $\mathrm{DCB}$ were combined in individual animals if both regions were obtained in that animal.

Analysis of the spatiotemporal structure of ongoing activity. For the analysis of ongoing activity phase coherence, we first calculated the mean ongoing activity trace for each tonotopic bin of areas AI, DC, and belt. Wavelet phase-coherence analysis between two ongoing activity traces was performed according to published methods (Lachaux et al., 1999; Le Van Quyen and Bragin, 2007). Briefly, each trace was first bandpass filtered (FIR filter; $\pm 0.5 \mathrm{~Hz}$ around the center frequency, length of four cycles) for the wavelet frequency of interest (i.e., $4 \mathrm{~Hz}$, unless otherwise indicated; we note that omission of this bandpass filtering step did not affect the results). Each filtered trace was then convolved with a $4 \mathrm{~Hz}$ Morlet wavelet (the Morlet wavelet was composed of 12 cycles). Following the convolution, we could derive the instantaneous phase and magnitude of each trace for the $4 \mathrm{~Hz}$ band of activity. To obtain the "phase-locking value" between two traces, a vector summation was performed for the resulting phase difference between the two traces across all time points (Lachaux et al., 1999). This value represents how consistent, on average, the phase difference is between two traces across time for a given frequency band, and ranges from 0 (not consistent) to 1 (perfectly consistent). This value is used as the measure of "phase coherence" or "temporal coherence" throughout the study. For calculating a phase-coherence map (see Fig. 4), the phase-locking value was calculated in this manner 
A
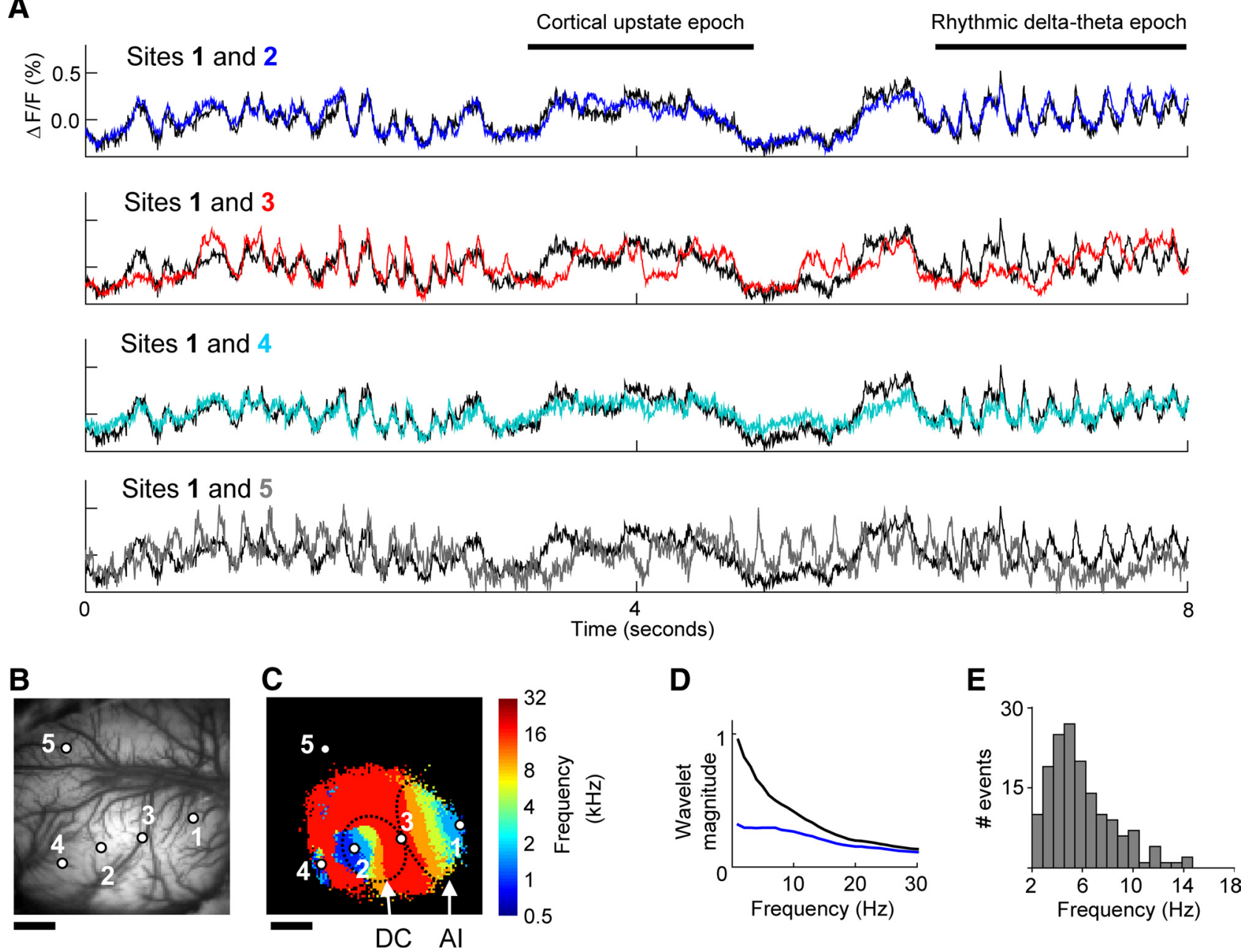

Figure 3. Ongoing slow-wave spontaneous activity recorded across functionally defined regions of several auditory cortical fields. $A$, Ongoing activity measured during silence across auditory and nonauditory cortex. Black trace, in all four plots, is from the low-frequency region of core area Al ( $2 \times 2$ pixels, centered on region " 1 " in B and C). Blue trace (region "2") is from low-frequency region of core area DC. Red trace (region " 3 ") is from the high-frequency region at the border of areas Al and DC. Cyan trace (region " 4 ") is from a low-frequency region of belt area VCB. Gray trace (region " 5 ") is from a region not responsive to the acoustic stimulus. $\boldsymbol{B}$, Image of cortical vasculature within the $10 \times 10 \mathrm{~mm}$ imaging region, with the five example region locations indicated. $\boldsymbol{C}$, The tonotopic map measured from this imaging region. $\boldsymbol{D}$, Wavelet amplitude spectra calculated from ongoing activity during silence (black) or during acoustic stimulation (blue, stimulus as in Fig. 1A), averaged over all auditory cortex core tonotopic bins from all animals. The wavelet analysis was performed as described in the Materials and Methods, but at wavelet frequencies ranging from 1 to $30 \mathrm{~Hz}$. $\boldsymbol{E}$, Histogram of interevent intervals for rhythmic event epochs from all animals, revealing a peak in the delta-theta range. Scale bars: $\boldsymbol{B}, \boldsymbol{C}, 2 \mathrm{~mm}$.

between a selected single pixel and all remaining $(100 \times 100)$ pixels of the imaging region.

Derivation of a significance level for ongoing activity phase coherence. We estimated a significance level for $4 \mathrm{~Hz}$ phase coherence between two ongoing activity traces. Since the $4 \mathrm{~Hz}$ Morlet wavelet was composed of 12 cycles ( $3 \mathrm{~s}$ in total), we assumed for this statistical test that phasedifference measurements separated by $3 \mathrm{~s}$ were independent. In this manner, we obtained 350 independent phase-difference samples (from each pair of tonotopic bins) from the spontaneous activity recorded across animals. Our measure of phase coherence (i.e., the phase-locking value) corresponds to the vector magnitude following a vector summation of phase-difference samples. Thus, a significant phase-coherence value corresponds to a distribution of circular phase-difference values that is nonuniform. Using the Rayleigh statistic for circular uniformity, we found that a vector magnitude (or phase-coherence value) of 0.11 corresponds to the $p=0.01$ significance level (when $N=350$ ). We verified that a permutation analysis performed on the experimentally measured phases gives the same significance level estimate. The $4 \mathrm{~Hz}$ phase-coherence values obtained between all pairs of tonotopic bins of AI and DC (which were the same whether they were estimated using all phase-difference samples or the subset of samples separated by $3 \mathrm{~s}$ ) were greater than this chance value of 0.11 .
Definition of delta-theta and upstate epochs. While spontaneous activity consisted of a variety of events, two prominently occurring event types with distinct and stereotypical temporal characteristics were rhythmic "deltatheta" events and cortical "upstates." Rhythmic delta-theta events were defined as high-amplitude ( $>0.1 \%$ valley-to-peak $\Delta F / F$ value in at least one tonotopic bin of a core or belt region) and short-duration $(\Delta F / F$ remained above a threshold value for $<100 \mathrm{~ms}$ ) events. Such events could occur in isolation, but often occurred in a series. The interevent interval within a given series was not perfectly constant, but the average interval peaked in the range of 3-6 Hz, as shown in Figure 3. In contrast, spontaneous upstates were defined as epochs during which the activity in a tonotopic bin of any cortical area remained, for at least $200 \mathrm{~ms}$ (some upstates lasted $>1 \mathrm{~s}$ ), above a threshold value. To determine this threshold, we first calculated the SD of the fluorescence value for each bin across the entire block. The upstate threshold was defined as $1 \mathrm{SD}$ above a stable local baseline $\Delta F / F$ value.

\section{Results}

Acoustic spectral tuning across multiple cortical fields Understanding the relationship between ongoing activity patterns and neuronal tuning requires a method capable of sampling neuronal activity over a large area, but with high spatial and 
temporal resolution. Voltage-sensitive dye imaging (VSDI) provides this combination of properties (Shoham et al., 1999). Previous work has established that the VSDI signal, in vivo, measures the aggregate subthreshold membrane potential from elements within the superficial cortical layers (Petersen et al., 2003a; Ferezou et al., 2007; Lippert et al., 2007). Ongoing activity measured with VSDI also resembles the local field potential signal in some aspects (Ferezou et al., 2007), although the mechanisms underlying the two signals are different. To characterize the bestfrequency tuning across multiple auditory cortical areas in the guinea pig, we employed VSDI during a continuous multitone sound stimulation paradigm. This consisted of pseudo-randomly presented pure-tone pips with frequencies ranging across six octaves (Fig. 1A). Fluorescence was sampled continuously during this paradigm (Fig. $1 B$ ) from an array of $100 \times 100$ imaging elements, which covered a $5 \times 5 \mathrm{~mm}$ area of cortex (Fig. $1 D$ ).

We found that the VSDI signal responded reliably to this multitone stimulus, allowing the rapid construction of frequencyspecific acoustic receptive fields from individual imaging pixels (Fig. 1C). Color coding each pixel according to the sound frequency eliciting the maximal response revealed the tonotopic organization of the region, with different pixels responding to the full range of frequencies known to be represented in guinea pig auditory cortex (Fig. 1E). The two tonotopically organized core auditory cortical fields of the guinea pig are visible in Figure $1 E$, namely, AI as well as the additional core area (DC). The layout of these areas is consistent with descriptions from previous electrophysiological studies (Redies et al., 1989b; Wallace et al., 2000), and the maps include a broader range of represented sound frequencies relative to previous VSDI studies (Horikawa et al., 2001; Nishimura et al., 2007). The best-frequency identity of pixels in the core fields was later used to explore the relationship between ongoing activity patterns and neuronal tuning.

Pixels sampling from regions outside of the auditory cortex core (i.e., auditory belt areas) responded less well to the multitone stimulus, perhaps because of differences across auditory cortical areas in temporal response properties (Eggermont, 1998). On the other hand, by presenting single pure-tone stimuli intermittently, we found reliable responses in both the core fields as well as a subset of belt areas (Redies et al., 1989b; Wallace et al., 2000). Restricted activation regions were observed within AI and DC at early latencies following stimulation with a low- or highfrequency pure-tone stimulus (Fig. $2 A, D$; a $10 \times 10 \mathrm{~mm}$ area of cortex was imaged in this case). At later latencies, activation spread within the core regions, and cortical belt areas were additionally recruited (Fig. $2 B, E$ ). These results indicate that belt fields can be distinguished from core areas both spatially and by their response latencies. The belt areas include the caudally located DCB and VCB (Fig. 2I). Additional belt fields are visible in this example and may correspond to rostral belt fields described by Wallace et al. (2000); however, these fields were not analyzed further because the cortical region containing them was only exposed in a subset of animals. Within the caudal belt fields, the location of initial activation was slightly offset in response to low-versus high-frequency tones (Fig. $2 B, E$ ). Indeed, pixels at the corresponding locations showed a bias for lower- versus higher-frequency stimuli (Fig. 2C,F). Thus, while pixels in these belt fields responded to a broader range of frequencies relative to pixels sampling from the core areas (Fig. 1), we nevertheless observed systematic biases in frequency tuning in the DCB and VCB areas and a coarse gradient of preferred frequencies.

\section{Ongoing slow-wave spontaneous activity patterns across auditory cortical fields}

We next examined the spatiotemporal structure of ongoing spontaneous activity across these functionally defined auditory cortical fields. Activity traces measured from local regions with VSDI during silence revealed two prominent spontaneous event types. The first are referred to as rhythmic delta-theta events, defined as short and sharp depolarization events that most often occurred rhythmically between the delta and theta ranges (3-6 $\mathrm{Hz}$; Fig. $3 A, E)$. The second are referred to as "cortical upstates," defined as prolonged ( $>200 \mathrm{~ms}$ ) depolarization events (Fig. $3 A$ ). The overall spectrum of the spontaneous activity following a wavelet analysis showed a falloff in amplitude with increasing temporal frequency, consistent with a pink-noise structure (Fig. $3 D)$. Together, these properties are consistent with the expected temporal structure of cortical spontaneous neural activity under anesthesia, as shown previously with electrophysiological techniques (Steriade et al., 1993; DeWeese and Zador, 2006; Chauvette et al., 2011).

We next visually compared ongoing spontaneous activity traces derived from auditory cortical and surrounding fields. We found that the pattern of activity in the auditory cortex was dramatically different than activity from an adjacent region that did not respond to acoustic stimuli (Fig. $3 A$, sites 1 and 5). Thus, consistent with recent reports, the cortical modality was an important factor for predicting the structure of slow-wave spontaneous activity (Mohajerani et al., 2010; Nir et al., 2011). More interestingly, however, the spatial resolution of VSDI permitted examining the pattern of spontaneous activity relative to acoustic spectral tuning properties across the auditory modality. Visual inspection revealed that subthreshold spontaneous activity was nonuniform across the auditory cortex and that distance was not the only factor determining its structure. For example, two pixels that were distant but had similar acoustic receptive fields (sampling from the low-frequency regions of areas AI and DC, respectively, separated by $\sim 5 \mathrm{~mm}$ ) had a very similar pattern of ongoing spontaneous activity (Fig. $3 A$, sites 1 and 2). A similar pattern was also observed for the low-frequency regions of AI and DCB areas, which are separated by $\sim 7 \mathrm{~mm}$ (Fig. $3 A$, sites 1 and 4 ). In contrast, two pixels that were nearer but had dissimilar acoustic receptive fields (sampling from low- and high-frequency regions of primary auditory cortex, respectively, separated by $\sim 2 \mathrm{~mm}$ ) had a more dissimilar pattern of activity (Fig. $3 A$, sites 1 and 3 ).

The visual inspection described so far suggests that neuronal tuning within the auditory modality could be an important factor in determining the structure of slow-wave ongoing activity. To illustrate this quantitatively, we calculated the degree of coupling (i.e., phase coherence) during ongoing spontaneous activity between a reference, or seed, pixel and all other pixels of the imaged region, covering core as well as belt fields. The spatial pattern of ongoing activity coherence could then be directly related to the sound-evoked maps obtained from the same region. We chose a wavelet phase-coherence method that describes the constancy of the phase relationship, in specific frequency bands, between two signals over time. We focused our analysis on a $4 \mathrm{~Hz}$ wavelet, given the prominence of this intrinsic rhythm in our recordings, and because it falls within a spectral range proposed to play important roles in auditory processing.

For example, we seeded the ongoing activity phase-coherence calculation on a pixel from the low best-frequency region of core area DC (Fig. 4C). We found that although high coherence was observed between this pixel and nearby pixels, as expected, a highly nonuniform pattern in coherence strength was observed 
across the imaged region. Coherence fell to a low value near the border between areas AI and DC, which represents high frequencies, but became very strong again at the other end of area AI where low frequencies are represented. Overall, the spatial pattern of phase coherence between a low-frequency pixel and the rest of the region, obtained from ongoing activity during silence, showed a striking resemblance to the pattern of activity evoked by a lowfrequency tone, across core as well as belt areas (Fig. 4, compare $C, A$ ). This illustrates that a low-frequency region of core auditory cortex has the highest temporal coherence during ongoing spontaneous activity, with a noncontiguous network of other low-frequency responsive regions throughout the auditory cortical modality. An analogous result was observed when the phase-coherence calculation was seeded on a high-frequency pixel of core auditory cortex (Fig. 4, compare $D$, $B)$. Finally, the region that was not responsive to auditory stimulation had high internal coherence, but low coherence with nearby regions that were sound responsive (Fig. 4E,F). Thus, while distance could play some role in determining coherence, revealing the fine functional architecture of the region predicted well the overall spatial phase-coherence pattern during ongoing slow-wave spontaneous activity.

To illustrate more completely the dynamics of ongoing activity across the tonotopic axis of core auditory cortex, we grouped pixels of areas AI and DC according to their best frequency into one of six one-octave-sized bins and displayed the average spontaneous activity traces over time for each bin (Fig. 5A). In other words, the two-dimensional map was reduced to one dimension by collapsing the iso-frequency axis. The resulting image represents the tonotopic-temporal pattern of activity during an $8 \mathrm{~s}$ epoch of silence. This image reveals that the two major spontaneous event types, namely, delta-theta events and cortical upstates, could be restricted to a portion of the tonotopic axis of auditory cortex at a given time. Further, a mirror image activation pattern is apparent across the tonotopic axis for individual events, indicating that activation occurs congruently for tonotopically matched subregions of areas AI and DC (Fig. 5A).

We quantified the pattern of coherence, relative to acoustic spectral tuning, by computing the phase coherence in delta-theta (i.e., $4 \mathrm{~Hz}$ ) ongoing activity between each pair of tonotopic bins from AI and DC. A matrix representation of the results indicates that phase coherence was always highest between pixels of AI and DC that had a similar best-frequency preference regardless of their relative cortical positions (Fig. $5 B$ ). Cross sections of the matrix illustrate the pattern of coherence when the calculation was seeded on example tonotopic bins from core auditory cortex (Fig. 5C, black lines in successive panels are from the first, middle, or bottom row of the matrix of Fig. $5 B$ ). For example, the lowestfrequency region of AI had higher coherence with the lowestfrequency region of DC than with an intermediately located region of high-frequency preference (Fig. $5 C$, black trace in first panel; coherence between AI bin 1 and DC bin 1, vs coherence between AI bin 1 and AI/DC bin 6, signed-rank test, $p<0.01$ ).
Similarly, a middle-frequency region of AI had higher coherence with a middle-frequency region of DC than with an intermediately located region of high-frequency preference (coherence between AI bin 3 and DC bin 3, vs coherence between AI bin 3 and AI/DC bin 6, signed-rank test, $p<0.01$; trace not shown). These results indicate quantitatively that tonotopy per se, independent of cortical distance, is an organizing factor for determining slow-wave activity phase coherence across the auditory cortex core.

A similar dependence of ongoing activity phase coherence on tonotopic identity was found for other slow-wave frequencies, whereas the effects became increasingly weaker for frequency bands higher than $\sim 8 \mathrm{~Hz}$ (Fig. 5D). The phase-coherence difference between bins of matching (AI bin 1 and DC bin 1) versus nonmatching (AI bin 1 and AI/DC bin 6) frequency preference was significantly greater than zero at wavelet frequencies of $1,2,4$, 8 , and $16 \mathrm{~Hz}$ (signed-rank test, $p<0.01$ ) but not $32 \mathrm{~Hz}$ (signedrank test, NS).

We note that although phase coherence of $4 \mathrm{~Hz}$ ongoing activity was higher between matched than between nonmatched frequency bins of AI and DC, the level of phase coherence was greater than chance between all pairs of bins (Rayleigh test, $p<$ 0.01 ; see Materials and Methods). Thus, phase coherence was above chance between each pair of bins of AI and DC, but tonotopic similarity had a significant additional effect.

We next asked whether a similar conclusion held separately for both major classes of spontaneous events observed in this study, namely, delta-theta events and cortical upstates, by examining correlations in activity over short time epochs containing only one event type or the other. We used a linear correlation method for this analysis instead of a wavelet method, given that cortical upstates are not oscillatory events. The analysis indicated that for both rhythmic delta-theta and upstate event epochs, correlations were highest for tonotopically congruent bins of fields 
A

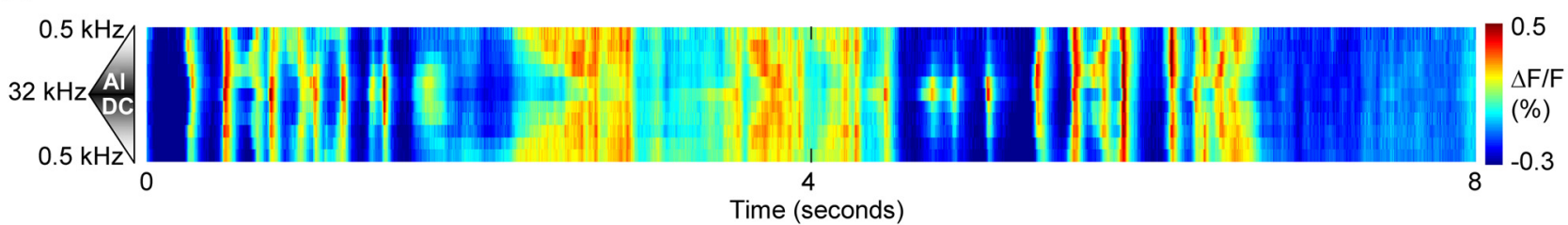

B

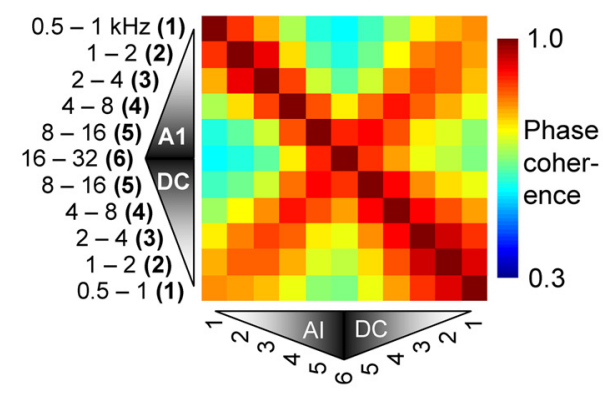

C

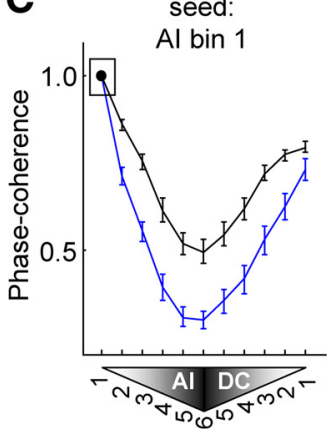

seed:

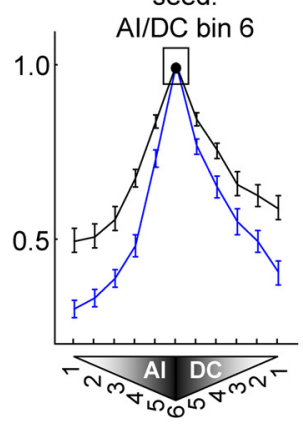

seed:

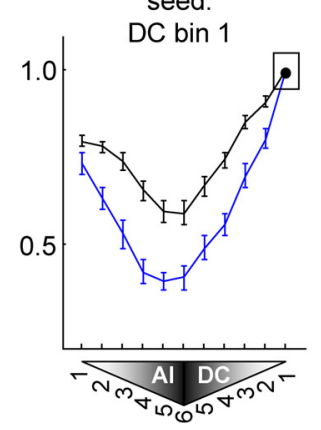

D

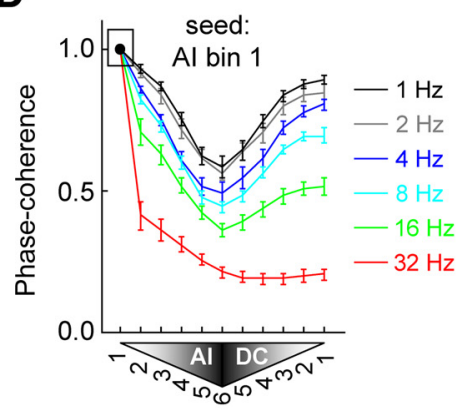

E

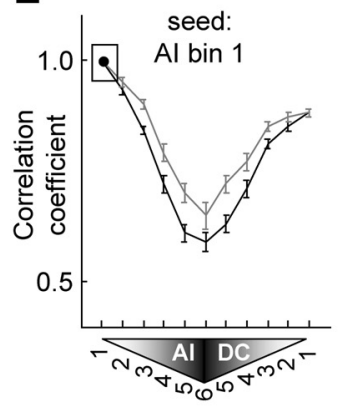

F

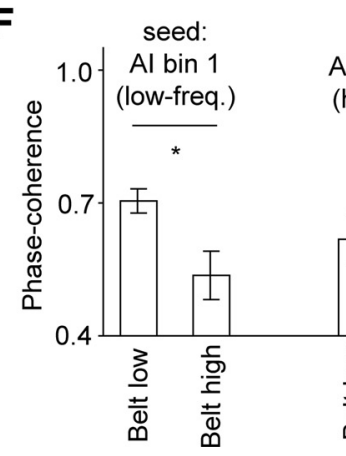

seed: Al/DC bin 6 (high-freq.) (low-freq.)

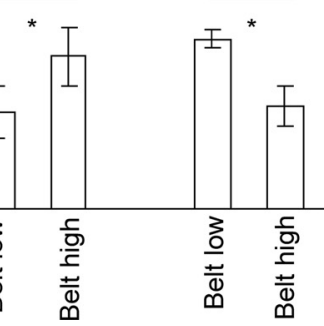

Figure 5. Ongoing activity phase-coherence quantification across core and belt auditory cortex. $A$, Pixels in core auditory cortex areas Al and DC were grouped according to their sound-frequency preference into one of six one-octave-sized bins. These bins were ordered along the vertical dimension of the image to preserve the tonotopic order across Al and DC. The mean spontaneous activity over all pixels of each bin is plotted over time. $\boldsymbol{B}$, Pixels in Al or DC were grouped into one-octave sized bins as in $\boldsymbol{A}$. The order of the bins in each axis of the plot corresponds to the topographic representation order across Al and DC. The phase-coherence value during ongoing spontaneous activity (with a $4 \mathrm{~Hz}$ wavelet) between pixels of each pair of bins is indicated and represents the mean over all animals. C, Plots of the phase coherence between a given tonotopic seed bin of area Al (indicated by black box) and all other bins of areas Al and DC. Calculations are for ongoing activity either during silence (black trace) or during continuous acoustic stimulation (stimulus as in Fig. $1 A$, blue trace). The mean and SEM across animals are shown. $D$, Phase coherence of ongoing activity during silence between tonotopic bin 1 of Al and other bins, where the wavelet frequency was 1,2,4,8,16, or $32 \mathrm{~Hz}$ as indicated by the line color. $\boldsymbol{E}$, The linear correlation coefficient during ongoing activity between the low-frequency bin of area Al (indicated by black box) and all other tonotopic bins of Al and DC. Calculations represent the mean and SEM over all epochs of ongoing activity containing only either rhythmic delta-theta events ( $N=56$; gray trace) or cortical upstates $(N=132$; black trace). $\boldsymbol{F}$, Phase coherence during spontaneous activity between the tonotopic bin of core auditory cortex indicated, and either the low- or high-frequency region of the belt. The mean and SEM across animals are shown.

AI and DC (Fig. 5E; coefficient of correlation between AI bin 1 and DC bin 1, vs coefficient between AI bin 1 and AI/DC bin 6, signed-rank test, $p<0.001$ for delta-theta epochs and upstate epochs).

The tonotopic specificity of spontaneous activity also extended to the belt areas of the auditory cortical network. Lowfrequency regions of core cortex had higher phase coherence with low- than with high-frequency belt regions (Fig. 5F; AI bin 1 and low-belt vs AI bin 1 and high-belt, signed-rank test, $p<0.05$; DC bin 1 and low-belt vs DC bin 1 and high-belt, signed-rank test, $p<0.05)$. Conversely, high-frequency regions of core cortex had higher coherence with high- than with low-frequency belt regions (Fig. 5F; AI/DC bin 6 and high-belt vs AI/DC bin 6 and low-belt, signed-rank test, $p<0.05$ ). In summary, local regions of multiple core and belt areas with similar spectral preferences to acoustic stimuli had the highest phase coherence in ongoing slow-wave activity recorded during silence.
Effects of acoustic stimulation on ongoing activity patterns We next asked whether the pattern of ongoing activity phase coherence was sensitive to acoustic stimulation. We found that continuous acoustic stimulation (stimulus shown in Fig. 1A) caused a temporal desynchronization in ongoing activity, such that low-frequency ongoing oscillations were reduced in amplitude (Fig. 3D). Stimulation also caused a spatial desynchronization of ongoing activity such that a more rapid falloff of phase coherence with cortical distance was observed (Fig. 5C, blue lines). Specifically, the coherence between the low- and highfrequency regions of core auditory cortex was significantly reduced during sensory stimulation (Fig. 5C, blue line in first panel; coherence between AI bin 1 and AI/DC bin 6 in ongoing activity recorded during silence vs stimulation, signed-rank test, $p<$ $0.01)$. However, there was no effect of continuous acoustic stimulation on the high level of coherence between tonotopic bins with similar best-frequency preference (Fig. $5 C$, blue line in first 
A

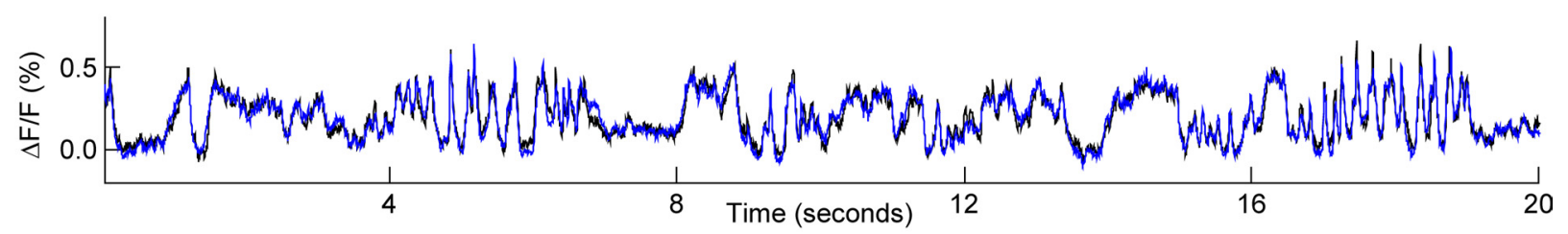

B

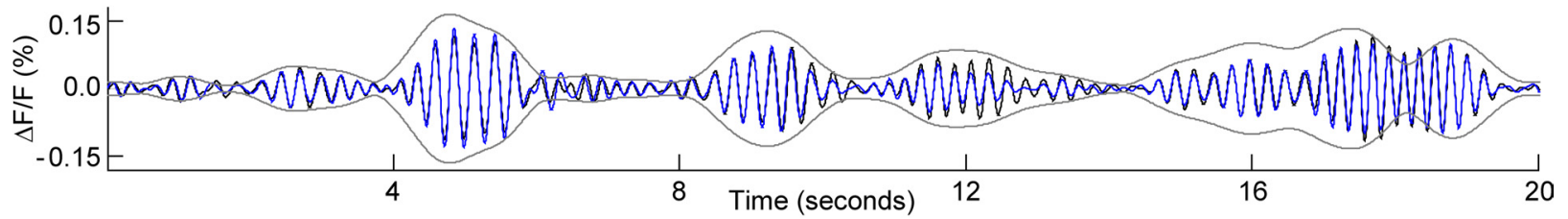

C

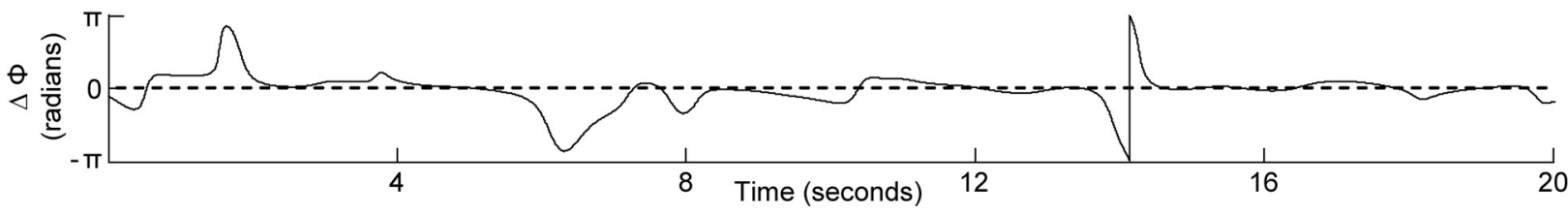

Figure 6. Dynamics of temporal coherence. $A$, Ongoing activity measured during silence, from the low-frequency region of core area $A I$ (black trace) and the low-frequency region of core area $D C$ (blue trace). $\boldsymbol{B}$, The corresponding ongoing activity traces from $\boldsymbol{A}$ are bandpass-filtered (FIR filter; $\pm 0.5 \mathrm{~Hz}$ around the center frequency of $4 \mathrm{~Hz}$, length of $1 \mathrm{~s}$ ) and the resulting waveforms plotted. Next, these signals were convolved with a $4 \mathrm{~Hz}$ Morlet wavelet. The resulting magnitudes were summed and correspond to the envelope that is plotted. $\boldsymbol{C}$, The instantaneous phase ( $\Phi$ ) difference between the traces from $\mathrm{Al}$ and $\mathrm{DC}$, following $4 \mathrm{~Hz}$ wavelet analysis.

panel; coherence between AI bin 1 and DC bin 1 in ongoing activity recorded during silence vs stimulation, signed-rank test, NS). The decreased phase coherence between cortical regions with different frequency preferences was expected, given that the presentation times of different frequencies in the multitone stimulus were uncorrelated. These results indicate that ongoing activity patterns in auditory cortex are sensitive to acoustic stimulation. Further, the pattern of desynchronization is broadly consistent with observations in the visual cortex indicating that increased visual drive reduces spatial correlations in ongoing activity (Kohn et al., 2009).

\section{Dynamics of coherence}

The previous results described time-averaged phase-coherence relationships between cortical regions, but given a sufficient signal-to-noise ratio in the signal, the dynamics of the phase relationships between the regions can also be appreciated. Figure $6 A$ shows ongoing activity recorded during silence from the lowfrequency regions of AI and DC, respectively (AI, black trace; DC, blue trace). Below this, the corresponding bandpass $(4 \pm 0.5 \mathrm{~Hz})$ filtered activity traces from the two regions are illustrated (Fig. $6 B)$. In addition, an envelope is plotted that indicates the summed magnitude of the signals following convolution with a (4 Hz) Morlet wavelet (Fig. 6B). High $4 \mathrm{~Hz}$ magnitudes (Fig. 6B) often correspond to times when delta-theta events are visible in the unfiltered traces (Fig. 6A). Figure $6 C$ illustrates the instantaneous value of the phase difference between the two signals following the wavelet convolution, and shows that a relatively constant phase difference is present between these two cortical regions of similar best-frequency preference. This constant phase difference is what gives rise to the high phase-coherence value between such regions. Comparison of panels $B$ and $C$ in Figure 6 indicates that the low- and constant-phase differences exist as long as moderate to high energy is present in the $4 \mathrm{~Hz}$ frequency band of the respective signals. Across animals, a similar pattern of dynamics was observed.

\section{Ongoing activity phase shifts between core and belt areas parallel evoked-activity latency differences}

The results presented thus far indicate that for slow-wave spontaneous activity, phase coherence is highest between regions that share best-frequency preference. This is due to a constant phase relationship between the signals recorded from the respective regions. However, a high phase coherence can reflect either similar phases between the two signals over time, or a phase shift between the signals that is constant over time. To distinguish between these alternatives and to better understand the mechanisms underlying the phase coherence, we computed the ongoing activity phase difference magnitudes between core and belt regions. Figure $7 A$ shows traces of ongoing activity simultaneously measured from three cortical areas with similar best-frequency preference, during an epoch with high delta-theta energy. Visual inspection suggests that phase differences might indeed be present between the activity traces. To explore this more completely, Figure $7 B$ shows the distribution of $4 \mathrm{~Hz}$ ongoing activity phase differences (across time and animals) between low-frequency regions of areas AI, DC, and the belt. The phase difference relationships are quantified in Figure $7 C$ (black symbols). Here, the mean phase difference (in radians) across time measured from each animal was converted to a corresponding time difference (the full period of a $4 \mathrm{~Hz}$ oscillation corresponds to $250 \mathrm{~ms}$ ). The mean phase difference in ongoing activity between areas AI and DC was not different from zero (signed-rank test, NS). On the other hand, there was a statistically significant phase shift in ongoing activity between AI and the belt (signed-rank test, $p<0.05$ ), as well as between DC and the belt (signed-rank test, $p<0.05$ ). Indeed, the phase differences between either core field (AI or DC) and the belt were greater than the phase differences between the two core fields (Kruskal-Wallis ANOVA, $p<0.01$; post hoc Mann-Whitney tests: AI-DC vs AI-belt, $p<0.01$; AI-DC vs DC-belt, $p<0.01$; AI-belt vs DC-belt, NS). We verified that an ongoing activity phase shift was similarly present between the 
A

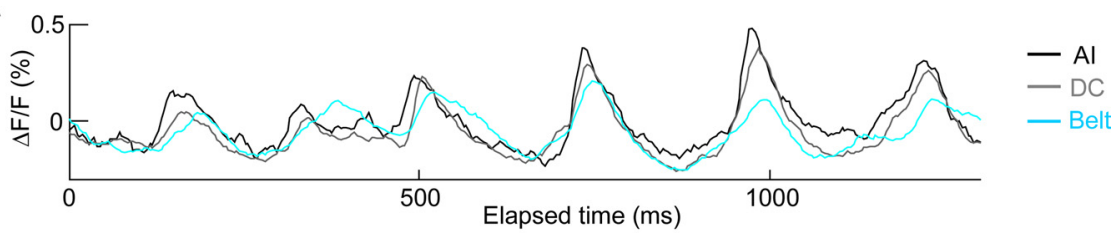

B

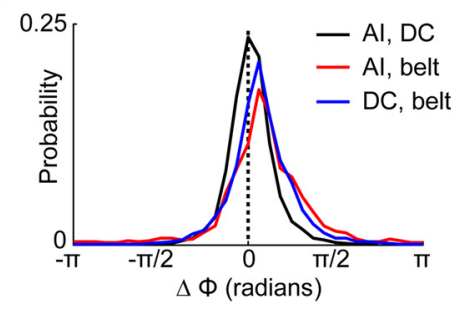

C

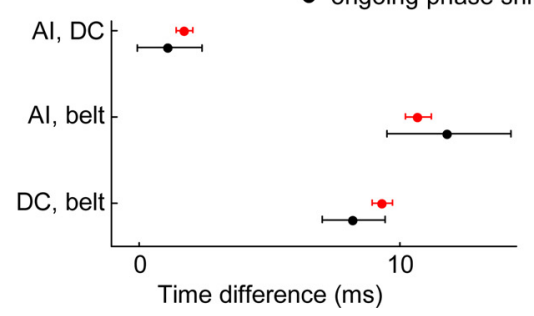

Figure 7. Temporal relationships between ongoing and evoked activity measured from core and belt areas. $\boldsymbol{A}$, Ongoing spontaneous activity measured simultaneously from low best-frequency regions of Al (black trace), DC (gray trace), and the belt area VCB (cyan trace) during an epoch of high delta-theta band activity. $\boldsymbol{B}$, Histograms of instantaneous phase $(\Phi)$ differences present in the $4 \mathrm{~Hz}$ band of ongoing spontaneous activity between $\mathrm{Al}$ and $\mathrm{DC}$, Al and belt, or DC and belt. The instantaneous phase differences were summed across all times in each animal, and across all animals. $C$, The mean phase difference across time in the $4 \mathrm{~Hz}$ band of ongoing spontaneous activity between each pair of regions was determined for each animal, and converted to milliseconds. From the same pairs of regions, the onset latency difference for activity elicited by pure-tone stimulation was measured. Mean \pm SEM across animals are shown.

high-frequency regions of the core and belt (signed-rank test, $p<0.05)$.

What is the origin and impact of the observed phase shift in ongoing activity between the core and the belt? We reasoned that the phase shift could relate to known latency differences between the core and belt areas for the arrival of sensory-evoked activity (Redies et al., 1989b; Wallace et al., 2000). To explore this possibility, we measured the latency of activity evoked by pure tones from the same core and belt areas (Fig. $7 C$, red symbols). As expected, we found that the difference in evoked-activity latency was greater between either core field (AI or DC) and the belt, compared with between the two core fields (Kruskal-Wallis ANOVA, $p<0.01$; post hoc Mann-Whitney tests: AI-DC vs AIbelt, $p<0.01$; AI-DC vs DC-belt, $p<0.01$; AI-belt vs DC-belt, $p<0.05$ ). Interestingly, however, for each pair of regions considered the ongoing activity phase differences (converted to timing differences) were not different from the latency differences for the arrival of evoked activity (Mann-Whitney tests comparing ongoing phase differences vs evoked latency differences: AI-DC, AI-belt, and DC-belt, NS). This indicates that the phase differences present in intrinsic ongoing activity between the core and belt regions parallel latency differences for evoked activity, suggesting that an evoked event may be expected to arrive at similar delta-theta band excitability phases across these areas.

\section{Discussion}

\section{Structure of ongoing slow-wave activity across the auditory cortical network}

The structure of ongoing activity informs how it may interact with stimulus-evoked activity to influence sensory processing (Kohn et al., 2009; Ringach, 2009). Our study focused on slowwave activity in auditory cortex given its distinct proposed roles, relative to spiking activity, in sensory and cognitive aspects of acoustic processing (Luo and Poeppel, 2007; Schroeder and Lakatos, 2009; Kayser et al., 2009; Shamma et al., 2011). We find that phase coherence in ongoing slow-wave activity is highest

between cortical regions with matched frequency preference. Further, ongoing activity is phase shifted between core and belt areas, but these phase shifts match latency differences for evoked activity. These data indicate that intrinsic slowwave events can modulate excitability in a spatially (i.e., neuronal tuning-dependent) and temporally coordinated manner across auditory cortical areas. We discuss the implications of these observations for underlying circuitry and acoustic processing below, but first relate them to previous studies of auditory cortex spontaneous activity.

The relationship between patterns in spontaneous activity and neuronal tuning has been previously examined in auditory cortex, but not for slow-wave activity. Most recently, recordings in awake macaques revealed that fluctuations of highgamma $(60-250 \mathrm{~Hz})$ range spontaneous activity were related to patterns of neuronal tuning across core and belt auditory cortical areas (Fukushima et al., 2012). This is consistent with previous observations within a single auditory cortical field, showing that correlations between neurons in spontaneous spiking-activity relate to similarities in neuronal tuning (Brosch and Schreiner, 1999; Noreña and Eggermont, 2003; Rothschild et al., 2010). None of these studies, which measured correlations in activity derived from single-unit- or multiunit-related recordings, addressed whether such relationships might be present in slowwave activity. A previous imaging study in primary auditory cortex also showed that the pattern of activity evoked by a pure tone was similar to patterns associated with a small subset of spontaneous events, although it is unclear how those events relate to the slow-wave activity studied here (Saitoh et al., 2010).

Several previous investigations, using simultaneous recordings of many single units, examined the temporal patterns of activation across local populations of neurons during cortical upstates, a type of slow-wave event also observed in our recordings. Those studies showed that spontaneous firing motifs observed across neurons within a local region were stereotyped and resembled motifs evoked by natural sounds (Luczak et al., 2009). Given the local sampling, whether the spontaneous motifs related to systematic variations in neuronal tuning across auditory cortex could not be examined. However, it was inferred that at a larger scale, the upstates were associated with traveling waves of activity (Luczak et al., 2007). Our wide-field imaging results support the notion that cortical upstates in auditory cortex, in some cases, are associated with propagating waves of activity (Fig. $5 A$ ). Interestingly, however, the waves do not propagate randomly across the auditory cortical modality, but rather propagate symmetrically across matched-frequency regions of multiple auditory cortical fields.

The relationship we find between the pattern of neuronal tuning and the pattern of slow-wave activity phase coherence resembles the relationship reported between neuronal tuning patterns and spontaneous fluctuations in high-gamma band activity, the latter of which is thought to reflect multiunit activity (Fukushima et al., 2012). Given the coupling between slow-wave activity phase and multiunit activity amplitude, our findings raise the 
possibility that the correlations measured in that study may relate to, or even result from, structure present in slow-wave activity (Lakatos et al., 2005; Schroeder and Lakatos, 2012).

The anesthetic agents used in this study impact some spatiotemporal aspects of ongoing activity, but seem unlikely to account for the relationship we observed between slow-wave ongoing activity patterns and functional tuning. Studies in the visual cortex have shown that anesthesia suppresses highfrequency energy and alters spatial correlations in spontaneous activity (Greenberg et al., 2008; Haider et al., 2013). On the other hand, the dependence of spontaneous activity correlation patterns in visual cortex on functional tuning has been observed under several different anesthesia regimens (Tsodyks et al., 1999; Kenet et al., 2003; Nauhaus et al., 2009; Ch'ng and Reid, 2010). In the auditory cortex, patterned spontaneous firing motifs across neuronal populations have been observed during cortical upstates in both anesthetized and quiet-awake conditions (Luczak et al., 2009). Further, the relationship alluded to above between spontaneous high-gamma activity fluctuation patterns and neuronal tuning in auditory cortex was measured in awake macaques (Fukushima et al., 2012). We suggest that the relationships observed in our study between slow-wave activity patterns and neuronal tuning are most likely accounted for by properties intrinsic to the circuits mediating the activity. The contexts during which this slow-wave activity may be recruited in awake, behaving animals remain to be defined (see below).

\section{Circuits underlying patterns in slow-wave ongoing activity}

Neurons within auditory cortex receive numerous sources of input that have the potential to influence spontaneous activity, and the circuits responsible for generating and propagating slowwave activity remain debated. The spatiotemporal patterns we observe can be compared to properties of anatomical circuits, to constrain underlying mechanisms. Several previous studies of spontaneous activity patterns within single auditory cortical fields have emphasized the contribution of local cortical circuits (Luczak et al., 2007; Luczak et al., 2009; Sakata and Harris, 2009; Saitoh et al., 2010). In contrast, the specific patterns observed for slow-wave activity in this study invoke additional essential roles for long-range connections in propagating slow-wave activity.

The specific projection from the sensory relay nucleus of the thalamus [ventral medial geniculate nucleus (vMGN)] is a primary candidate for relaying the tonotopically matched patterns of activity across the core auditory fields (AI and DC) observed in this study for both spontaneous delta-theta events and cortical upstates. However, several pieces of evidence suggest additional participating circuits. First, the vMGN does not appear to project to belt fields (Redies et al., 1989a) and thus cannot explain the high levels of phase coherence that exist between core and belt fields. Second, the phase delays present in delta-theta band ongoing activity between the core and belt fields also argue against the possibility that a single common long-range projection is responsible for their coherence. Thus, circuits in addition to the projection from the vMGN are necessary for mediating high phase coherence between core and belt fields. One probable substrate is the direct corticocortical projections between tonotopically organized core and belt auditory areas, which are a conserved feature of auditory cortex across mammalian species (Schreiner and Winer, 2007). These projections link regions with similar bestfrequency preference preferentially, although not exclusively. An alternative substrate, which could explain high phase coherence between core and belt areas, is a recently identified circuit linking the primary and secondary areas of cortex via the thalamus, al- though the tonotopic specificity of this circuit has not been addressed (Theyel et al., 2010).

In contrast to the set of circuits we invoke for explaining the observed specific patterns of phase coherence, other candidate circuits seem less probable. For example, while the matrix of calbindin-positive cells in thalamus projects throughout core and belt auditory areas (Jones, 2001), the properties of this projection seem inconsistent with explaining the tonotopic specificity we observe for ongoing activity patterns between core and belt fields, and with the phase shifts present in the activity between core and belt fields. This projection may mediate more diffuse patterns in slow-wave activity. In particular, it may contribute to a higher level of phase coherence present within, relative to between, cortical sensory modalities (Mohajerani et al., 2010; Nir et al., 2011).

The central role we invoke for the vMGN in projecting the observed slow-wave activity patterns from the thalamus to the core fields of auditory cortex is consistent with an emerging view of slow-wave activity generation and its dependence on a thalamo-cortical-thalamic loop (Crunelli and Hughes, 2010; Ushimaru et al., 2012). This circuit consists centrally of the thalamic reticular nucleus, sensory relay nuclei of the thalamus (the vMGN in this case), and input and output layers of cortex, the latter of which feed back to thalamic nuclei. Anatomical data suggest that connections between each of the nodes in this network can be tonotopically specific (Kimura et al., 2012). This circuit thus has the potential to generate specific patterns of slowwave activity, which would be propagated to the core auditory cortical fields via the vMGN.

Slow-wave ongoing activity patterns and auditory processing The spatial and temporal properties of intrinsic ongoing activity inform not only how this activity is produced, but how it may interact with evoked activity to shape sensory processing. Auditory cortical ongoing activity in the delta-theta range is reliably modulated by natural acoustic stimuli, and approximately represents the slow temporal envelope of the acoustic input (Luo and Poeppel, 2007; Kayser et al., 2009; Chandrasekaran et al., 2010; Szymanski et al., 2011). Intrinsic factors can further modulate this representation. For example, attending to a rhythmic stimulus alters the phase or temporal coherence of delta-theta range ongoing activity (Lakatos et al., 2008; Elhilali et al., 2009; Xiang et al., 2010). This intrinsic modulation of temporal coherence is hypothesized to enhance the representation of an attended rhythmic input. Schroeder and Lakatos (2009) have proposed that this rhythmic modulation may occur within specific representation regions, in the case of feature-specific attention. While this spatial specificity has not yet been demonstrated, an analogous process has been observed, albeit for high-frequency oscillations, in the visual modality. During visual selective-attention tasks, enhanced coherence within high-frequency bands is observed in spatially restricted subregions of multiple cortical fields (Gregoriou et al., 2009).

It is useful to consider what circuits could contribute to attention processes in the auditory modality, and whether a signal could act in a coordinated manner across the multiple core and belt areas recruited during auditory cortical processing. The view from previous studies that intrinsic subthreshold slow-wave rhythms are diffuse traveling waves (Volgushev et al., 2006; Ferezou et al., 2007; Harris and Thiele, 2011) is consistent with their playing a role in synchronizing processing over large cortical territories (Jones, 2001), but not in enhancing specific representations within those regions. Our results, on the other hand, indicate that intrinsic slow-wave rhythms can modulate excit- 
ability across distributed auditory fields in a tonotopically specific manner. These properties of slow-wave rhythms and their generating circuits may impact on acoustic processing under particular circumstances; for example, when attending to a specific acoustic input. In support of this possibility, the same circuitry that could account for the slow-wave activity patterns observed here, which includes the thalamic reticular nucleus (Crunelli and Hughes, 2010; Ushimaru et al., 2012), is also involved in attention or gating processes (McAlonan et al., 2006; Zikopoulos and Barbas, 2006; McAlonan et al., 2008; Yu et al., 2009).

\section{References}

Arieli A, Sterkin A, Grinvald A, Aertsen A (1996) Dynamics of ongoing activity: explanation of the large variability in evoked cortical responses. Science 273:1868-1871. CrossRef Medline

Brosch M, Schreiner CE (1999) Correlations between neural discharges are related to receptive field properties in cat primary auditory cortex. Eur J Neurosci 11:3517-3530. CrossRef Medline

Busch NA, Dubois J, VanRullen R (2009) The phase of ongoing EEG oscillations predicts visual perception. J Neurosci 29:7869-7876. CrossRef Medline

Chandrasekaran C, Turesson HK, Brown CH, Ghazanfar AA (2010) The influence of natural scene dynamics on auditory cortical activity. J Neurosci 30:13919-13931. CrossRef Medline

Chauvette S, Crochet S, Volgushev M, Timofeev I (2011) Properties of slow oscillation during slow-wave sleep and anesthesia in cats. J Neurosci 31: 14998-15008. CrossRef Medline

Ch'ng YH, Reid RC (2010) Cellular imaging of visual cortex reveals the spatial and functional organization of spontaneous activity. Front Integr Neurosci 4:20. CrossRef Medline

Crunelli V, Hughes SW (2010) The slow $(<1 \mathrm{~Hz})$ rhythm of non-REM sleep: a dialogue between three cardinal oscillators. Nat Neurosci 13: 9-17. CrossRef Medline

deCharms RC, Blake DT, Merzenich MM (1998) Optimizing sound features for cortical neurons. Science 280:1439-1443. CrossRef Medline

DeWeese MR, Zador AM (2006) Non-Gaussian membrane potential dynamics imply sparse, synchronous activity in auditory cortex. J Neurosci 26:12206-12218. CrossRef Medline

Eggermont JJ (1998) Representation of spectral and temporal sound features in three cortical fields of the cat. Similarities outweigh differences. J Neurophysiol 80:2743-2764. Medline

Elhilali M, Xiang J, Shamma SA, Simon JZ (2009) Interaction between attention and bottom-up saliency mediates the representation of foreground and background in an auditory scene. PLoS Biol 7:e1000129. CrossRef Medline

Ferezou I, Haiss F, Gentet LJ, Aronoff R, Weber B, Petersen CC (2007) Spatiotemporal dynamics of cortical sensorimotor integration in behaving mice. Neuron 56:907-923. CrossRef Medline

Fukushima M, Saunders RC, Leopold DA, Mishkin M, Averbeck BB (2012) Spontaneous high-gamma band activity reflects functional organization of auditory cortex in the awake macaque. Neuron 74:899-910. CrossRef Medline

Greenberg DS, Houweling AR, Kerr JN (2008) Population imaging of ongoing neuronal activity in the visual cortex of awake rats. Nat Neurosci 11:749-751. CrossRef Medline

Gregoriou GG, Gotts SJ, Zhou H, Desimone R (2009) High-frequency, long-range coupling between prefrontal and visual cortex during attention. Science 324:1207-1210. CrossRef Medline

Haider B, Häusser M, Carandini M (2013) Inhibition dominates sensory responses in the awake cortex. Nature 493:97-100. CrossRef Medline

Harris KD, Thiele A (2011) Cortical state and attention. Nat Rev Neurosci 12:509-523. CrossRef Medline

Horikawa J, Hess A, Nasu M, Hosokawa Y, Scheich H, Taniguchi I (2001) Optical imaging of neural activity in multiple auditory cortical fields of guinea pigs. Neuroreport 12:3335-3339. CrossRef Medline

Jones EG (2001) The thalamic matrix and thalamocortical synchrony. Trends Neurosci 24:595-601. CrossRef Medline

Kayser C, Montemurro MA, Logothetis NK, Panzeri S (2009) Spike-phase coding boosts and stabilizes information carried by spatial and temporal spike patterns. Neuron 61:597-608. CrossRef Medline

Kenet T, Bibitchkov D, Tsodyks M, Grinvald A, Arieli A (2003) Spontane- ously emerging cortical representations of visual attributes. Nature 425 : 954-956. CrossRef Medline

Kimura A, Yokoi I, Imbe H, Donishi T, Kaneoke Y (2012) Auditory thalamic reticular nucleus of the rat: anatomical nodes for modulation of auditory and cross-modal sensory processing in the loop connectivity between the cortex and thalamus. J Comp Neurol 520:1457-1480. CrossRef Medline

Kohn A, Zandvakili A, Smith MA (2009) Correlations and brain states: from electrophysiology to functional imaging. Curr Opin Neurobiol 19:434438. CrossRef Medline

Lachaux JP, Rodriguez E, Martinerie J, Varela FJ (1999) Measuring phase synchrony in brain signals. Hum Brain Mapp 8:194-208. CrossRef Medline

Lakatos P, Shah AS, Knuth KH, Ulbert I, Karmos G, Schroeder CE (2005) An oscillatory hierarchy controlling neuronal excitability and stimulus processing in the auditory cortex. J Neurophysiol 94:1904-1911. CrossRef Medline

Lakatos P, Karmos G, Mehta AD, Ulbert I, Schroeder CE (2008) Entrainment of neuronal oscillations as a mechanism of attentional selection. Science 320:110-113. CrossRef Medline

Le Van Quyen M, Bragin A (2007) Analysis of dynamic brain oscillations: methodological advances. Trends Neurosci 30:365-373. CrossRef Medline

Lippert MT, Takagaki K, Xu W, Huang X, Wu JY (2007) Methods for voltage-sensitive dye imaging of rat cortical activity with high signal-tonoise ratio. J Neurophysiol 98:502-512. CrossRef Medline

Luczak A, Barthó P, Marguet SL, Buzsáki G, Harris KD (2007) Sequential structure of neocortical spontaneous activity in vivo. Proc Natl Acad Sci U S A 104:347-352. CrossRef Medline

Luczak A, Barthó P, Harris KD (2009) Spontaneous events outline the realm of possible sensory responses in neocortical populations. Neuron 62:413425. CrossRef Medline

Luo H, Poeppel D (2007) Phase patterns of neuronal responses reliably discriminate speech in human auditory cortex. Neuron 54:1001-1010. CrossRef Medline

McAlonan K, Cavanaugh J, Wurtz RH (2006) Attentional modulation of thalamic reticular neurons. J Neurosci 26:4444-4450. CrossRef Medline

McAlonan K, Cavanaugh J, Wurtz RH (2008) Guarding the gateway to cortex with attention in visual thalamus. Nature 456:391-394. CrossRef Medline

Mohajerani MH, McVea DA, Fingas M, Murphy TH (2010) Mirrored bilateral slow-wave cortical activity within local circuits revealed by fast bihemispheric voltage-sensitive dye imaging in anesthetized and awake mice. J Neurosci 30:3745-3751. CrossRef Medline

Nauhaus I, Busse L, Carandini M, Ringach DL (2009) Stimulus contrast modulates functional connectivity in visual cortex. Nat Neurosci 12 : 70-76. CrossRef Medline

Ng BS, Schroeder T, Kayser C (2012) A precluding but not ensuring role of entrained low-frequency oscillations for auditory perception. J Neurosci 32:12268-12276. CrossRef Medline

Nir Y, Staba RJ, Andrillon T, Vyazovskiy VV, Cirelli C, Fried I, Tononi G (2011) Regional slow waves and spindles in human sleep. Neuron 70: 153-169. CrossRef Medline

Nishimura M, Shirasawa H, Kaizo H, Song WJ (2007) New field with tonotopic organization in guinea pig auditory cortex. J Neurophysiol 97:927932. CrossRef Medline

Noreña AJ, Eggermont JJ (2003) Changes in spontaneous neural activity immediately after an acoustic trauma: implications for neural correlates of tinnitus. Hear Res 183:137-153. CrossRef Medline

Petersen CC, Grinvald A, Sakmann B (2003a) Spatiotemporal dynamics of sensory responses in layer $2 / 3$ of rat barrel cortex measured in vivo by voltage-sensitive dye imaging combined with whole-cell voltage recordings and neuron reconstructions. J Neurosci 23:1298-1309. Medline

Petersen CC, Hahn TT, Mehta M, Grinvald A, Sakmann B (2003b) Interaction of sensory responses with spontaneous depolarization in layer $2 / 3$ barrel cortex. Proc Natl Acad Sci U S A 100:13638-13643. CrossRef Medline

Redies H, Brandner S, Creutzfeldt OD (1989a) Anatomy of the auditory thalamocortical system of the guinea pig. J Comp Neurol 282:489-511. CrossRef Medline

Redies H, Sieben U, Creutzfeldt OD (1989b) Functional subdivisions in the 
auditory cortex of the guinea pig. J Comp Neurol 282:473-488. CrossRef Medline

Ringach DL (2009) Spontaneous and driven cortical activity: implications for computation. Curr Opin Neurobiol 19:439-444. CrossRef Medline

Rothschild G, Nelken I, Mizrahi A (2010) Functional organization and population dynamics in the mouse primary auditory cortex. Nat Neurosci 13:353-360. CrossRef Medline

Saitoh K, Inagaki S, Nishimura M, Kawaguchi H, Song WJ (2010) Spontaneous activity resembling tone-evoked activity in the primary auditory cortex of guinea pigs. Neurosci Res 68:107-113. CrossRef Medline

Sakata S, Harris KD (2009) Laminar structure of spontaneous and sensoryevoked population activity in auditory cortex. Neuron 64:404-418. CrossRef Medline

Schreiner CE, Winer JA (2007) Auditory cortex mapmaking: principles, projections, and plasticity. Neuron 56:356-365. CrossRef Medline

Schroeder CE, Lakatos P (2009) Low-frequency neuronal oscillations as instruments of sensory selection. Trends Neurosci 32:9-18. CrossRef Medline

Schroeder CE, Lakatos P (2012) The signs of silence. Neuron 74:770-772. CrossRef Medline

Shamma SA, Elhilali M, Micheyl C (2011) Temporal coherence and attention in auditory scene analysis. Trends Neurosci 34:114-123. CrossRef Medline

Shoham D, Glaser DE, Arieli A, Kenet T, Wijnbergen C, Toledo Y, Hildesheim R, Grinvald A (1999) Imaging cortical dynamics at high spatial and temporal resolution with novel blue voltage-sensitive dyes. Neuron 24:791-802. CrossRef Medline

Stefanics G, Hangya B, Hernádi I, Winkler I, Lakatos P, Ulbert I (2010) Phase entrainment of human delta oscillations can mediate the effects of expectation on reaction speed. J Neurosci 30:13578-13585. CrossRef Medline
Steriade M, Nuñez A, Amzica F (1993) Intracellular analysis of relations between the slow $(<1 \mathrm{~Hz})$ neocortical oscillation and other sleep rhythms of the electroencephalogram. J Neurosci 13:3266-3283. Medline

Szymanski FD, Rabinowitz NC, Magri C, Panzeri S, Schnupp JW (2011) The laminar and temporal structure of stimulus information in the phase of field potentials of auditory cortex. J Neurosci 31:15787-15801. CrossRef Medline

Theyel BB, Llano DA, Sherman SM (2010) The corticothalamocortical circuit drives higher-order cortex in the mouse. Nat Neurosci 13:84-88. CrossRef Medline

Tsodyks M, Kenet T, Grinvald A, Arieli A (1999) Linking spontaneous activity of single cortical neurons and the underlying functional architecture. Science 286:1943-1946. CrossRef Medline

Ushimaru M, Ueta Y, Kawaguchi Y (2012) Differentiated participation of thalamocortical subnetworks in slow/spindle waves and desynchronization. J Neurosci 32:1730-1746. CrossRef Medline

Volgushev M, Chauvette S, Mukovski M, Timofeev I (2006) Precise longrange synchronization of activity and silence in neocortical neurons during slow-wave oscillations. J Neurosci 26:5665-5672. CrossRef Medline

Wallace MN, Rutkowski RG, Palmer AR (2000) Identification and localisation of auditory areas in guinea pig cortex. Exp Brain Res 132:445-456. CrossRef Medline

Xiang J, Simon J, Elhilali M (2010) Competing streams at the cocktail party: exploring the mechanisms of attention and temporal integration. J Neurosci 30:12084-12093. CrossRef Medline

Yu XJ, Xu XX, He S, He J (2009) Change detection by thalamic reticular neurons. Nat Neurosci 12:1165-1170. CrossRef Medline

Zikopoulos B, Barbas H (2006) Prefrontal projections to the thalamic reticular nucleus form a unique circuit for attentional mechanisms. J Neurosci 26:7348-7361. CrossRef Medline 